\title{
Covariant derivative expansion of fermionic effective action at high temperatures
}

\author{
Dmitri Diakonov ${ }^{\text {* }}$ and Michaela Oswald \\ ${ }^{a}$ NORDITA, Blegdamsvej 17, DK-2100 Copenhagen, Denmark \\ ${ }^{b}$ NBI, Blegdamsvej 17, 2100 Copenhagen, Denmark
}

(Dated: April 30, 2004)

\begin{abstract}
We derive the contribution of massless fermions to the 1-loop effective action for static $A_{4}$ and $A_{i}$ fields at high temperatures, for the $S U(2)$ gauge group assuming that gluon fields are slowly varying but allowing for an arbitrary amplitude of $A_{4}$.

PACS numbers: 11.15.-q,11.10.Wx,11.15.Tk

Keywords: gauge theories, finite temperature field theory, derivative expansion
\end{abstract}

\section{INTRODUCTION}

Quantum chromodynamics (QCD) at non-zero temperature is an intensely studied field. At very high temperatures the coupling constant is small and perturbation theory can be developed. However, due to the chromomagnetic sector of the theory, perturbation theory explodes already at a few-loop level [1, 2, 3] and is hence only applicable at academically high temperatures [4]. The region of intermediate temperatures is of much bigger interest. Both the restoration of chiral symmetry and the deconfinement are believed to take place in this region. In the presence of fermions it is still unclear if there is a confinement-deconfinement phase transition or just a smooth crossover between the two phases. In any case QCD is in the deconfined plasma phase at very high temperatures.

At finite temperature gluons obey periodic and fermions obey anti-periodic boundary conditions in imaginary time. This property leads to the quantized Matsubara frequencies. They are even multiples of $\pi T$ for gluons, i.e. $\omega_{k}=2 k \pi T$, and odd multiples of $\pi T$ for quarks, i.e. $\omega_{k}=(2 k+1) \pi T$. So while gluons have a zero mode this is not the case for quarks. This fact has direct and important influence on the IR behavior of the two contributions to the effective action.

At the tree level very heavy modes decouple from a theory at high temperatures. This is called dimensional reduction [5] since the heavy modes are simultaneously the time-dependent ones. Neglecting all modes except the zero Matsubara frequencies leaves a 3D static theory

$$
-\frac{1}{4 g^{2}} F_{\mu \nu}^{2}+\psi_{f}^{\dagger} i \not \nabla \psi_{f} \rightarrow-\frac{1}{4 g^{2} T}\left[F_{i j}^{2}+2\left(D_{i}^{a b} A_{4}^{b}\right)^{2}\right]
$$

which only contains the static gluonic modes with the coupling constant $g_{3}^{2}=g^{2} T$. Since the energy can never vanish for fermions they decouple completely.

The long-range forces mediated by the static gluons lead to the IR divergencies, because in strict perturbation

\footnotetext{
*Electronic address: diakonov@nordita.dk
}

${ }^{\dagger}$ Electronic address: oswald@alf.nbi.dk theory they are massless. Fermions do not cause any IR problems, since they do not have zero modes even if they are massless, which is the case we consider here. Nevertheless, fermions change the effective action in a drastic way as compared to the pure glue case, since their presence changes the symmetry of the action with respect to the center-of-group gauge transformations.

The tree-level action (11) has certainly insufficient accuracy to study field fluctuations at high but not infinitely high temperatures. Once one includes quantum corrections both the fermions and all the non-zero Matsubara modes of the gluons show up in the loops.

Effective theories resulting from quantum corrections for different energy scales, $T, g T$ and $g^{2} T$ have been constructed in [6, 7]. The parameters in the effective theories are obtained by matching the correlation functions between the effective and the actual theory as functions of the parameters of the original theory. The question of color-conductivity and transport properties of the plasma has been addressed in [8]. A heat kernel approach for Yang-Mills theories has been used in [9], a constraint effective potential for the Polyakov loop has been studied in [10] and spatial variations of the Polyakov loop have been investigated in 11].

In the pure Yang-Mills theory the center symmetry plays a crucial part in the description of the the confinement-deconfinement phase transition 11, 12, 13, 14]. The latter is usually characterized by an order parameter which is the average of the trace of the so-called Polyakov line:

$$
P(x)=\mathrm{P} \exp \left(i \int_{0}^{1 / T} d x_{4} A_{4}\right) .
$$

The order parameter $<\operatorname{Tr} P>$ is zero in the confined phase below the critical temperature and assumes a nonzero value in the deconfined phase above the critical temperature. The Polyakov line is not invariant under gauge transformations belonging to the gauge group center. One hence concludes that if $\langle\operatorname{Tr} P>=0$ then the $Z\left(N_{c}\right)$ symmetry is manifest. This situation describes confinement. If for any reason $<\operatorname{Tr} P>\neq 0$ then the symmetry must have been broken. This corresponds to the deconfined phase.

The 1-loop [3, 15, 16] and 2-loop [17] potential ener- 
gies as functions of $A_{4}$ are known. They are periodic functions of the eigenvalues of $A_{4}$ in the adjoint representation with period $2 \pi T$. This reflects the symmetry of the $Z\left(N_{c}\right)$ vacua. The curvature of the potential at its minima gives the leading order Debye mass for 'electric' gluons. The zero energy minima of the potential correspond to quantized values of $A_{4}$ or center group values for the Polyakov line, where $\operatorname{Tr} P \neq 0$. At high temperatures the system oscillates around one of these minima. At lower temperatures, however, the fluctuations around the minimum increase and eventually the system undergoes a phase transition to $\langle\operatorname{Tr} P>=0$. At the same time, one expects that near the phase transition point the fluctuations are long-range. To study those fluctuations, one needs an effective low-momenta theory which, however, does not assume that the $A_{4}$ component is small.

Let us formulate the problem more mathematically. Nonzero temperatures explicitly break the $4 D$ Euclidean symmetry of the theory down to the $3 D$ Euclidean symmetry, so that the spatial $A_{i}$ and time $A_{4}$ components of the Yang-Mills field play different roles and should be treated differently. One can always choose a gauge where $A_{4}$ is time-independent. Taking $A_{4}(x)$ to be static is not a restriction of any kind on the fields but merely a convenient gauge choice, and we shall imply this gauge throughout the paper. [It is also a possible gauge choice at $T=0$ but in that limiting case it is unnatural as one usually wishes to preserve the $4 D$ symmetry.] As to the spatial components $A_{i}(x, t)$, they are, generally speaking, time-dependent, although periodic in the time direction. Putting the components $A_{i}$ to zero is a gauge non-invariant restriction on the fields since any time-independent gauge transformation will generate a nonzero $A_{i}$. Therefore, the spatial derivatives of the Polyakov line in the gauge-invariant effective action can only appear as covariant derivatives including a nonzero $A_{i}$ field.

In [18] we calculated the 1-loop kinetic energy for the eigenvalues of the Polyakov line, integrating over gluon and ghost fluctuations. See also [19] for a summary. In this work we are interested in obtaining the effect of quarks on that kinetic energy as well. We use a background field method for the gluons and evaluate the 1loop action through a functional determinant formalism. In particular we assume the background fields to vary slowly but the $A_{4}$ component is allowed to have an arbitrary amplitude. We integrate out fast varying quantum fluctuations about them by making an expansion in spatial covariant derivatives. This method was originally developed in [20] for zero temperature QCD.

This corresponds to summing up all powers of $A_{4}$ but where their momenta are restricted to $p<T$ reflecting the long-range behavior of the plasma phase. As we said, we choose a static gauge for $A_{4}(x)$. This gauge choice does not prevent $A_{i}(x)$ from being time dependent. Since $A_{i}\left(x_{4}, x\right)$ is periodic in time, its time derivative is given by the Matsubara frequencies $\omega_{k}=2 \pi k T$ being $O(T)$ for any $k \neq 0$. Since we are interested in low momenta fluctuations, $p<T$, it is consistent to restrict oneself to the zero Matsubara frequency of the background field, i.e. to the static $A_{i}(x)$.

We expect that our results are suitable to study the correlation functions of the Polyakov line not too far from the transition point where it experiences fluctuations that are large in amplitude but presumably mainly long ranged. The results may be of some help for studying quantum weights of semiclassical objects, such as dyons $(21,22])$ or calorons 23.

The effective action contains a contribution from the gluons and from the fermions. The former part, namely the pure Yang-Mills effective action, was obtained by the authors in [18]. Although, as discussed above, there is no center symmetry for the fermions, it is still instructive to see their effect on the effective action for the Polyakov line. In particular we work with the gauge group $\mathrm{SU}(2)$, We consider a general electric field but restrict ourselves to a magnetic field parallel to $A_{4}, B_{i}^{\|}$. The expected result for the effective action, which is the sum of the tree-level and 1-loop actions, is hence:

$$
\begin{aligned}
{\left[S_{\mathrm{eff}}^{\mathrm{F}}\right]^{(2)}=} & \int \frac{d^{3} x}{T}\left[-T^{3} V^{\mathrm{F}}\left(A_{4}^{2}\right)\right. \\
& +E_{i}^{2} F_{1}^{(\mathrm{F})}\left(A_{4}^{2}\right)+\frac{\left(E_{i} A_{4}\right)^{2}}{A_{4}^{2}} F_{2}^{(\mathrm{F})}\left(A_{4}^{2}\right) \\
& \left.+\left(B_{i}^{\|}\right)^{2} H_{1}^{(\mathrm{F})}\left(A_{4}^{2}\right)+\ldots\right]
\end{aligned}
$$

with the electric and magnetic fields

$$
\begin{aligned}
& E_{i}^{a}=D_{i}^{a b} A_{4}^{b}-\dot{A}_{i}^{a}=\partial_{i} A_{4}^{a}+\epsilon^{a c b} A_{i}^{c} A_{4}^{b}-\dot{A}_{i}^{a}, \\
& B_{i}^{a}=\frac{1}{2} \epsilon_{i j k}\left(\partial_{j} A_{k}^{a}-\partial_{k} A_{j}^{a}+\epsilon^{a b c} A_{j}^{b} A_{k}^{c}\right) .
\end{aligned}
$$

The first term is the potential energy and the remaining terms are the kinetic energy contributions in the colorelectric and color-magnetic sector. The objective of this paper is to find these functions.

\section{THE QCD ACTION AT FINITE TEMPERATURE}

The basics about Yang-Mills theory at finite temperature were discussed in [18]. The (Yang-Mills) action of gluons at finite temperature is given by

$$
S^{\mathrm{YM}}=\int_{0}^{\beta} d x_{4} \int d^{3} x\left[-\frac{1}{4 g^{2}(M)} F_{\mu \nu}^{a} F_{\mu \nu}^{a}\right], \quad \beta=\frac{1}{T},
$$

where the gluon fields obey periodic boundary conditions in the temporal direction, i.e.

$$
A_{\mu}(0, x)=A_{\mu}(\beta, x)
$$

Because of the compactified time direction there is a group of special gauge transformations which transform the gluon fields in the usual way as

$$
A_{\mu} \rightarrow U A_{\mu} U^{-1}+i U \partial_{\mu} U^{-1}
$$


and which preserve the periodicity condition (6), but which are not periodic themselves:

$$
U(0, x)=z_{k} U(\beta, x)
$$

Here $z_{k}$ is an element of the center group $Z\left(N_{c}\right)$ :

$$
z_{k}=e^{2 \pi i k / N_{c}} \quad k \in\left\{0, N_{c}-1\right\}
$$

The Yang-Mills action is invariant under this gauge transformation, but the Polyakov line is not. It transforms as

$$
P(x)=\mathrm{P} \exp \left(i \int_{0}^{1 / T} d t A_{4}\right) \rightarrow z_{k}^{-1} P(x)
$$

From this property one sees immediately that the $Z\left(N_{c}\right)$ symmetry implies $<\operatorname{Tr} P>=0$ while it must be (spontaneously) broken if $\langle\operatorname{Tr} P>\neq 0$.

Including $N_{f}$ quarks with mass $m_{f}$ the full QCD action at finite temperature becomes

$$
\begin{aligned}
S & =\int_{0}^{\beta} d x_{4} \int d^{3} x \\
& \times\left[-\frac{1}{4 g^{2}(M)} F_{\mu \nu}^{a} F_{\mu \nu}^{a}+\sum_{f=1}^{N_{f}} \psi_{f}^{\dagger}\left(i \not \nabla+i m_{f}\right) \psi_{f}\right],
\end{aligned}
$$

where the Dirac operator is given by

$$
i \not \nabla=i \not \partial+T^{a} A_{\mu}^{a} \gamma^{\mu}
$$

Here the $T^{a}$ are the generators of $S U(N)$ in their fundamental representation, they are half the Pauli matrices for $\mathrm{SU}(2)$. The fermions in eq. (11) obey anti-periodic boundary conditions

$$
\psi_{f}(0, x)=-\psi_{f}(\beta, x)
$$

This property is, however, not preserved by the $Z\left(N_{c}\right)$ gauge transformation (8). Specifically the fermions transform as

$$
\begin{aligned}
\psi_{f}^{U}(\beta, x) & =U(\beta, x) \psi_{f}(\beta, x) \\
\psi_{f}^{U}(0, x) & =U(0, x) \psi_{f}(0, x)=z_{k} U(\beta, x) \psi_{f}(0, x) \\
& =-z_{k} U(\beta, x) \psi_{f}(\beta, x)=-z_{k} \psi_{f}^{U}(\beta, x) .
\end{aligned}
$$

Hence in the presence of fermions the $Z\left(N_{c}\right)$ symmetry gets explicitly broken and the Polyakov line ceases to serve as an exact order parameter for the theory, since $<\operatorname{Tr} P>\neq 0$ for all temperatures. Nevertheless, even in the presence of massless fermions the Polyakov line might provide useful information near the critical temperature [24].

\section{ONE LOOP QUANTUM ACTION}

The partition function of QCD in its Euclidean invariant form is given by

$$
\begin{aligned}
& Z\left(A, \psi_{f}, \bar{\psi}_{f}\right)=\sum_{f=1}^{N_{f}} \int D A D \psi_{f} D \psi_{f}^{\dagger} \exp \int d^{4} x \\
& \quad \times\left[-\frac{1}{4 g^{2}(M)} F_{\mu \nu}^{a} F_{\mu \nu}^{a}+\sum_{f=1}^{N_{f}} \psi_{f}^{\dagger}\left(i \not \nabla+i m_{f}\right) \psi_{f}\right] .
\end{aligned}
$$

We use the background field method for the gluon fields, where we decompose them into background fields and quantum fluctuations around them which we assume to be small:

$$
A_{\mu}=\bar{A}_{\mu}+a_{\mu}
$$

In this work we are interested in a 1-loop effective theory for the background $\bar{A}$ fields. This corresponds to an expansion of the action around the background gluon fields to quadratic order in the quantum fluctuations $a_{\mu}$. The one loop expansion of the gluon Lagrangian is:

$$
\begin{aligned}
-\frac{1}{4 g^{2}(M)} F_{\mu \nu}^{2}(A)= & -\frac{1}{4 g^{2}(M)} F_{\mu \nu}^{2}(\bar{A}) \\
& -\frac{1}{g^{2}(M)} D_{\mu}(\bar{A}) F_{\mu \nu}(\bar{A}) a_{\nu} \\
& -\frac{1}{2 g^{2}(M)} a_{\mu}^{a} W_{\mu \nu}^{a b} a_{\nu}^{b}+\ldots
\end{aligned}
$$

where

$$
W_{\mu \nu}^{a b}=-\left[D^{2}(\bar{A})\right]^{a b} \delta_{\mu \nu}+\left[D_{\mu} D_{\nu}\right]^{a b}-2 f^{a c b} F_{\mu \nu}^{c}(\bar{A})
$$

and

$$
D_{\mu}^{a b}(\bar{A})=\partial_{\mu} \delta^{a b}+f^{a c b} \bar{A}_{\mu}^{c}
$$

is the covariant derivative in the background field in the adjoint representation. The second term in eq. (17), which is linear in $a_{\nu}$, is zero if the background field obeys the equation of motion. In the fermionic Lagrangian the quarks couple to the gluon fields in the usual minimal, i.e. linear way. Hence the expansion is just

$$
\psi_{f}^{\dagger} i \not \nabla \psi_{f}=\psi_{f}^{\dagger} i \not \nabla(\bar{A}) \psi_{f}+\psi_{f}^{\dagger} a_{\mu} \gamma_{\mu} \psi_{f}+\ldots
$$

where $\not \nabla(\bar{A})$ is the covariant derivative of the background field in the fundamental representation. The second term in eq. (20) contributes at the 2-loop level which we do not consider here.

The quadratic form $W_{\mu \nu}^{a b}$ in eq. (18) is degenerate: it has an infinite number of zero modes which are the infinitesimal gauge transformations $a_{\mu}^{a}=D_{\mu}^{a b} \Lambda^{b}$. In order to remove this degeneracy one has to fix the gauge for these fluctuations. We choose the background Lorenz gauge $D_{\mu}(\bar{A}) a_{\mu}=0$ [29]. The second term in eq. (18) 
cancels out but the Faddeev-Popov ghost determinant arises which again can be expressed as a Grassmann integral over ghost fields.

The 1-loop partition function thus becomes

$$
\begin{aligned}
& Z(\bar{A})=e^{\bar{S}} \int D a D \chi D \chi^{+} D \bar{\psi}_{f} D \psi_{f} \exp \left\{\int d^{4} x\right. \\
& \left.\times\left[-\frac{1}{2 g^{2}(M)}\left(a_{\mu}^{b} W_{\mu \nu}^{b c} a_{\nu}^{c}-\chi^{+a} D_{\mu}^{2} \chi^{a}\right)+\sum_{f=1}^{N_{f}} \psi_{f}^{\dagger} i \not D \psi_{f}\right]\right\},
\end{aligned}
$$

where $\chi, \chi^{+}$are ghost fields,

$$
i \not D_{f}=i \not \nabla+i m_{f}=i \not \partial+T^{a} \bar{A}_{\mu}^{a} \gamma^{\mu}+i m_{f}
$$

is the massive Dirac operator in the fundamental representation, and

$$
\bar{S}=-\frac{1}{4 g^{2}(M)} \int d^{4} x F_{\mu \nu}^{a}(\bar{A}) F_{\mu \nu}^{a}(\bar{A})
$$

is the action of the background gluon fields.

Integrating out the quarks, ghosts and the quantum fluctuations of the gluons leaves us with the desired effective theory for the background $\bar{A}$ fields:

$$
Z(\bar{A})=e^{\bar{S}}(\operatorname{det} W)^{-1 / 2} \operatorname{det}\left(-D^{2}\right) \prod_{f=1}^{N_{f}} \operatorname{det}\left(i \not D_{f}\right)
$$

so that the 1-loop action is

$$
\begin{aligned}
S_{1-\text { loop }} & =\log (\operatorname{det} W)^{-1 / 2}+\log \operatorname{det}\left(-D^{2}\right) \\
& +\sum_{f=1}^{N_{f}} \log \operatorname{det}\left(i \not_{f}\right) .
\end{aligned}
$$

Since the $\bar{A}$ are the only gluon fields left we will omit the bar from now on. So far the background field has been kept arbitrary. One has, however, the gauge freedom to choose the $A_{4}(x)$ fields to be static. The spatial gluon components are generally time dependent. Since $A_{i}\left(x_{4}, x\right)$ is periodic in time, its time derivative is given by the Matsubara frequencies $\omega_{k}=2 \pi k T$ being $O(T)$ for any $k \neq 0$. Since we are interested in low momenta fluctuations, $p<T$, we shall restrict ourselves to the zero Matsubara frequency of the background field, i.e. to the static $A_{i}(x)$.

The operators in the ghost and gluon functional determinants, $D^{2}$ and $W$, are matrices in the adjoint representation of the color group, and they are built from covariant derivatives and the field strength only. We used this fact in [18] to make an expansion of the 1-loop pure Yang-Mills action in powers of $D_{i}$. Since the (static) electric field is given by $E_{i}^{a}=D_{i}^{a b} A_{4}^{b}$ and the magnetic field by $B_{k}^{a}=\frac{1}{2} \epsilon_{i j k} F_{i j}^{a}=\frac{1}{4} \epsilon_{i j k} \epsilon^{c a d}\left[D_{i}, D_{j}\right]^{c d}$ we obtained an effective action for the background $A_{4}$ fields in terms of electric and magnetic fields.
In this paper we study the contribution of the fermion functional determinant to that effective action. We use again the technique of the covariant derivative expansion. In addition, we will work in the chiral limit throughout, i.e. we set $m_{f}=0$. The main difference to the gluon calculation is that in the case of fermions we are dealing with operators in the fundamental representation and that we do not expect $Z\left(N_{c}\right)$ symmetric results.

\section{THE FERMIONIC FUNCTIONAL DETERMINANT}

Throughout this paper we will be working with Euclidean coordinates. A summary of our conventions is given in the Appendix. In particular we use the following:

$$
\begin{aligned}
\left\{\gamma^{\mu}, \gamma^{\nu}\right\} & =2 \delta^{\mu \nu} \mathbf{1}_{\mathbf{4}} \\
{\left[\gamma^{\mu}, \gamma^{\nu}\right] } & =4 \mathrm{i} \sigma^{\mu \nu}
\end{aligned}
$$

where the $\gamma_{\nu}$ denote the Euclidean Dirac matrices, and $\sigma^{\mu \nu}$ are the spin matrices. Since we are working in the chiral limit the Dirac operator is given by eq. (12) and is by definition hermitian:

$$
i \not \nabla=i \not \partial+T^{a} A_{\mu}^{a} \gamma^{\mu}=(i \not \nabla)^{\dagger} .
$$

The covariant derivative defines the field strength tensor in the fundamental representation as

$$
\left[\nabla_{\mu}, \nabla_{\nu}\right]=-i F_{\mu \nu}
$$

The functional determinant of the fermions can be written as

$$
\operatorname{det}(i \not)=\sqrt{\operatorname{det}(i \not \nabla)(i \not)}
$$

which following a method originally introduced by Schwinger 26] can be further expressed as

$$
\operatorname{det}(i \not \nabla)=\exp \left(-\frac{1}{2} \operatorname{Sp} \int_{0}^{\infty} \frac{d s}{s} e^{-s(i \not \nabla)(i \not \nabla)}\right) .
$$

Here Sp is the functional trace. For its contribution to the effective action we have to properly normalize it, i.e. subtract the free zero-gluon part:

$$
\log \operatorname{det}(i \not \nabla)_{n}=-\frac{1}{2} \operatorname{Sp} \int_{0}^{\infty} \frac{d s}{s}\left(e^{s \not^{2}}-e^{s \not^{2}}\right) .
$$

The square of $\varnothing$ can be decomposed further,

$$
\not^{2}=\gamma_{\mu} \gamma_{\nu} \nabla_{\mu} \nabla_{\nu}=\frac{1}{2}\left(\left\{\gamma_{\mu}, \gamma_{\nu}\right\} \nabla_{\mu} \nabla_{\nu}+\left[\gamma_{\mu}, \gamma_{\nu}\right] \nabla_{\mu} \nabla_{\nu}\right)
$$

Since in the second term on the l.h.s. the commutator is antisymmetric we can also antisymmetrize

$$
\nabla_{\mu} \nabla_{\nu} \rightarrow \frac{1}{2}\left[\nabla_{\mu}, \nabla_{\nu}\right]
$$


With Eqs. (26 2729) one finds

$$
\nabla^{2}=\nabla^{2} \mathbf{1}_{\mathbf{4}}+\sigma_{\mu \nu} F_{\mu \nu} .
$$

Equation (32) hence becomes

$$
\begin{aligned}
& \log \operatorname{det}(i \not \nabla)_{n}=-\frac{1}{2} \operatorname{Sp} \int_{0}^{\infty} \frac{d s}{s} \\
& \quad \times\left\{\exp \left[s\left(\nabla^{2} \mathbf{1}_{\mathbf{4}}+\sigma_{\mu \nu} F_{\mu \nu}\right)\right]-\exp \left[s \partial^{2} \mathbf{1}_{\mathbf{4}}\right]\right\} .
\end{aligned}
$$

The 1-loop action is UV divergent. This comes from the fact that the running coupling constant is divergent at the tree level. Since QCD is a renormalizable theory, the tree level divergence has to be canceled by a 1-loop divergence. In order to control the divergent behavior of (34) we regularize the determinants by introducing a Pauli-Villars cutoff $\mathrm{M}$ in momentum space. This means that we use the so-called "quadrupole formula":

$$
\begin{aligned}
& \operatorname{det}(i \not)_{n, r}=\sqrt{\frac{\operatorname{det}\left(-\not \nabla^{2}\right)}{\operatorname{det}\left(-\not \partial^{2}\right)} \frac{\operatorname{det}\left(-\not \partial^{2}+M^{2}\right)}{\operatorname{det}\left(-\not \not^{2}+M^{2}\right)}} \\
& =\exp \left\{-\frac{1}{2} \int_{0}^{\infty} \frac{d s}{s} \operatorname{Sp}\left[\left(1-e^{s M^{2}}\right)\left(e^{s \not^{2}}-e^{s \not^{2}}\right)\right]\right\} .
\end{aligned}
$$

The functional trace Sp can be taken by inserting any complete basis. We choose the plane wave basis:

$$
\mathrm{Sp} e^{-s K}=\operatorname{Tr} \int d^{4} x \lim _{y \rightarrow x} \int \frac{d^{4} p}{(2 \pi)^{4}} e^{-i p \cdot y} e^{-s K} e^{i p \cdot x},
$$

where $\operatorname{Tr}$ is the remaining matrix trace over color and Lorentz indices. One can now drag the latter plane-wave exponent though the differential operator $K$ until it cancels with the former. This results in the shift of the derivatives inside the differential operator and in the following representation of the functional trace [20]:

$$
\operatorname{Sp} e^{-s K}=\operatorname{Tr} \int d^{4} x \int \frac{d^{4} p}{(2 \pi)^{4}} e^{-s K\left(\partial_{\alpha} \rightarrow \partial_{\alpha}+i p_{\alpha}\right)} \mathbf{1} .
$$

The $\mathbf{1}$ at the end is meant to emphasize that the shifted operator acts on unity, so that for example any term that has a $\partial_{\alpha}$ in the exponent and is brought all the way to the right, will vanish. According to (37) we now have

$$
\begin{aligned}
& \log \operatorname{det}(i \not \nabla)_{n, r}=-\frac{1}{2} \int d^{3} x \sum_{k=-\infty}^{\infty} \int \frac{d^{3} p}{(2 \pi)^{3}} \int_{0}^{\infty} \frac{d s}{s}\left(1-e^{-s M^{2}}\right) \\
& \times \operatorname{Tr}\left\{\exp \left[s\left(\nabla_{4}+i \omega_{k}\right)^{2} \mathbf{1}_{\mathbf{4}}+s\left(\nabla_{i}+i p_{i}\right)^{2} \mathbf{1}_{\mathbf{4}}+s \sigma_{\mu \nu} F_{\mu \nu}\right]\right. \\
& \left.-\exp \left[s\left(i \omega_{k}\right)^{2} \mathbf{1}_{\mathbf{4}}+s\left(i p_{i}\right)^{2} \mathbf{1}_{\mathbf{4}}\right]\right\} .
\end{aligned}
$$

Let us now define

$$
\mathcal{B} \equiv \nabla_{4}+i \omega_{k} \mathbf{1}_{\mathbf{2}}
$$

then eq. (38) becomes

$$
\begin{aligned}
& \log \operatorname{det}(i \not \nabla)_{n, r}=-\frac{1}{2} \int d^{3} x \sum_{k=-\infty}^{\infty} \int \frac{d^{3} p}{(2 \pi)^{3}} \\
& \times \int_{0}^{\infty} \frac{d s}{s} \operatorname{Tr} e^{-s p^{2}}\left(1-e^{-s M^{2}}\right)\left\{-e^{-s \omega_{k}^{2}}\right. \\
& \left.+\exp \left[\left(s \mathcal{B}^{2}+s \nabla_{i}^{2}+2 i s p_{i} \nabla_{i}\right) \mathbf{1}_{\mathbf{4}}+s \sigma_{\mu \nu} F_{\mu \nu}\right]\right\} .
\end{aligned}
$$

This result is independent of the gauge group. In the following we will work with $S U(2)$. In particular we choose the background $A_{4}$ fields to be a) static and b) diagonal, i.e.

$$
A_{4}(x)=\phi(x) \frac{\tau_{3}}{2}
$$

then

$$
\mathcal{B}=-i \phi(x) \frac{\tau_{3}}{2}+i \omega_{k} \mathbf{1}_{\mathbf{2}}
$$

Here $\tau_{3}$ is the third of the three Pauli matrices:

$$
\tau_{1}=\left(\begin{array}{ll}
0 & 1 \\
1 & 0
\end{array}\right), \quad \tau_{2}=\left(\begin{array}{cc}
0 & -i \\
i & 0
\end{array}\right), \quad \tau_{3}=\left(\begin{array}{cc}
1 & 0 \\
0 & -1
\end{array}\right) .
$$

\section{COVARIANT DERIVATIVE EXPANSION}

\section{A. Zeroth order - The fermionic potential}

In this order we set the spatial covariant derivative to zero, i.e. $\nabla_{i}=0$, but sum over all powers of $\nabla_{4}$ :

$$
\begin{aligned}
{\left[\log \operatorname{det}(i \not \nabla)_{n, r}\right]^{(0)}=} & -\frac{1}{2} \int d^{3} x \sum_{k=-\infty}^{\infty} \int \frac{d^{3} p}{(2 \pi)^{3}} \int_{0}^{\infty} \frac{d s}{s}(44) \\
& \times \operatorname{Tr} e^{-s p^{2}}\left(e^{s \mathcal{B}^{2} \mathbf{1}_{4}}-e^{s \omega_{k}^{2} \mathbf{1}_{4}}\right) .
\end{aligned}
$$

This can be evaluated explicitly. With eq. (42) it is easy to check that

$$
\operatorname{Tr} e^{s \mathcal{B}^{2} \mathbf{1}_{4}}=4\left[e^{-\frac{1}{4} s\left(\phi-2 \omega_{k}\right)^{2}}+e^{-\frac{1}{4} s\left(\phi+2 \omega_{k}\right)^{2}}\right],
$$

where the factor 4 comes from $\operatorname{Tr} \mathbf{1}_{\mathbf{4}}$. Since the fermionic energies are given by $\omega_{k}=(2 k+1) \pi T$ we can rewrite the terms in eq. (45) as

$$
e^{-\frac{1}{4} s\left(2 \omega_{k} \pm \phi\right)^{2}} \equiv e^{-s\left[2 \pi T k-\phi_{ \pm}\right]^{2}},
$$

where we defined

$$
\phi_{ \pm}=\frac{\phi}{2} \pm \pi T
$$

The summation over $\omega_{k}$ and the integrations over $s$ and $p$ can now be performed along the lines of the bosonic case ([18]), using the formula

$$
\sum_{k=-\infty}^{\infty} \int_{0}^{\infty} \frac{d s}{s} e^{-s(2 \pi T k-\tilde{\phi})^{2}-s p^{2}}=-\log \left(\operatorname{ch} \frac{|\vec{p}|}{T}-\cos \frac{\tilde{\phi}}{T}\right) .
$$

The result is the following:

$$
\begin{aligned}
& {\left[\log \operatorname{det}(i \not \nabla)_{n, r}\right]^{(0)}=\frac{1}{12 \pi^{2} T} \int d^{3} x} \\
& \times\left[\phi_{+}^{2}\left(2 \pi T-\phi_{+}\right)^{2}+\phi_{-}^{2}\left(2 \pi T-\phi_{-}\right)^{2}-2(\pi T)^{4}\right]_{\bmod 2 \pi T} \\
& =\frac{1}{96 \pi^{2} T} \int d^{3} x\left\{\left[\phi^{2}-(2 \pi T)^{2}\right]^{2}-(2 \pi T)^{4}\right\}_{\bmod 4 \pi T} .
\end{aligned}
$$




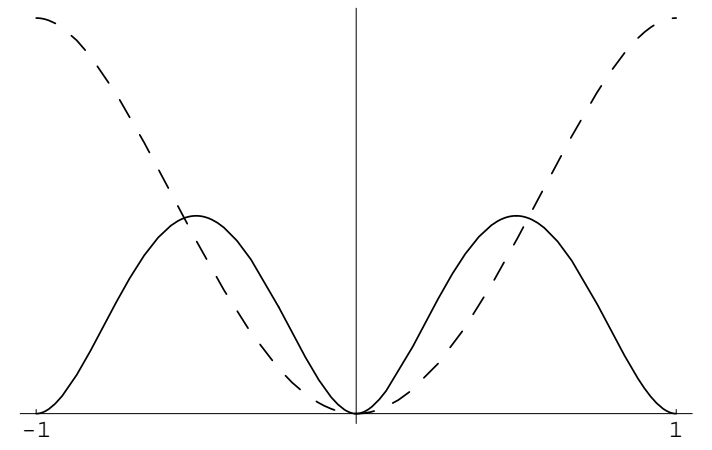

FIG. 1: The gluon (solid) and fermion (dashed) potentials for $N_{f}=1$ and $-1 \leq \nu \leq 1$.

This potential is symmetric around $\phi=0$ and is periodic with period $4 \pi T$ in contrast to the gluon potential (3], 15]) which has period $2 \pi T$. The curvature around $\phi=0$ of eq. (49) gives the fermionic contribution to $-m_{D}^{2} / T$. Indeed we find $-\left(N_{f} T\right) / 6$ which is in accordance with the known [3] 1-loop result for the Debye mass

$$
m_{D}^{2}=\frac{1}{3} T^{2}\left(N_{c}+\frac{N_{f}}{2}\right) .
$$

If we add the corresponding result from the gluons (see e.g. [18])

$$
\frac{1}{12 \pi^{2} T} \int d^{3} x\left[\phi^{2}(2 \pi T-\phi)^{2}\right] \mid \bmod 2 \pi T
$$

then we get the full result for two colors, namely $(2+$ $\left.N_{f} / 2\right) T / 3$. Introducing the variable $\nu=\phi /(2 \pi T)$ we have $\phi_{ \pm}=\pi T(\nu \pm 1)$ and eq. (49) becomes

$$
\left[\log \operatorname{det}(i \not \nabla)_{n, r}\right]^{(0)}=T^{3} \frac{\pi^{2}}{6} \int\left[d^{3} x\left(1-\nu^{2}\right)^{2}-1\right]_{\bmod 2} .
$$

The potential is then given by the (in the chiral case identical) contributions of all $N_{f}$ quark flavors:

$$
V^{\mathrm{F}}=-N_{f} \frac{(2 \pi)^{2}}{24}\left[\left(1-\nu^{2}\right)^{2}-1\right]_{\bmod 2} .
$$

This result is of course well known and can for example be found in the Appendix D of [] or in [16]. It can be compared to the pure Yang-Mills potential $([\underline{3}],[15])$

$$
V^{\mathrm{YM}}=\left.\frac{(2 \pi)^{2}}{3} \nu^{2}(1-\nu)^{2}\right|_{\bmod 1} .
$$

Both potentials are shown in Fig. [1]

We see clearly that the YM potential has $N_{c}$ minima, which are the $Z\left(N_{c}\right)$ symmetric points of $A_{4}=0$. Since there is no center symmetry for the fermions, we do not find the same situation. Indeed the fermion potential has a minimum at $\nu=0$ and a maximum at $\nu=1$. Its period is doubled relative to the YM potential. This fact comes solely from the fundamental representation of the fermions.

\section{B. Leading terms in electric sector}

For an effective theory we are interested in the leading terms in the electric sector. We would like to stress that we are keeping all powers of the background $A_{4}$ field in our approach, but make an expansion in the spatial covariant derivative. This means that we allow for an arbitrary amplitude of the $A_{4}$ fields but we assume that all the background fields are slowly varying and have momenta $p<T$. For the leading terms we hence expand to quadratic order in $\nabla_{i}$. Just as for the gluons and ghosts [18] the technique is to expand

$$
\operatorname{Tr} \exp \left\{s\left[\left(\mathcal{B}^{2}+\nabla_{i}^{2}+2 i p_{i} \nabla_{i}\right) \mathbf{1}_{\mathbf{4}}+\sigma_{\mu \nu} F_{\mu \nu}\right]\right\}
$$

in powers of $\nabla_{i}$ using the following two master formulas for two non-commuting matrices $A$ and $B$ :

$$
\begin{aligned}
e^{A+B}= & e^{A}+\int_{0}^{1} d \alpha e^{\alpha A} B e^{(1-\alpha) A} \\
& +\int_{0}^{1} d \alpha \int_{0}^{1-\alpha} d \beta e^{\alpha A} B e^{\beta A} B e^{(1-\alpha-\beta)} A+\ldots
\end{aligned}
$$

and

$$
\left[B, e^{A}\right]=\int_{0}^{1} d \gamma e^{\gamma A}[B, A] e^{(1-\gamma) A} .
$$

Since in eq. (56) powers of $B$ are brought down in the expansion we identify $B$ with the combinations of covariant derivatives in eq. (55) and $A$ is the rest. The electric field is identified as

$$
\left[\nabla_{i}, \mathcal{B}\right]=\left[\nabla_{i}, \nabla_{4}\right]=-i F_{i 4}=-i E_{i}=-i E_{i}^{a} T^{a} .
$$

To the second order in $\nabla_{i}$ there are three terms contributing:

$$
\begin{aligned}
T_{1}= & s \operatorname{Tr} \int_{0}^{1} d \alpha e^{\alpha s \mathcal{B}^{2} \mathbf{1}_{4}}\left(\nabla_{i}^{2} \mathbf{1}_{\mathbf{4}}\right) e^{(1-\alpha) s \mathcal{B}^{2} \mathbf{1}_{\mathbf{4}}} \\
T_{2}= & -\frac{4 p^{2} s^{2}}{3} \operatorname{Tr} \int_{0}^{1} d \alpha \int_{0}^{1-\alpha} d \beta e^{\alpha s \mathcal{B}^{2} \mathbf{1}_{4}}\left(\nabla_{i} \mathbf{1}_{\mathbf{4}}\right) \\
& \times e^{\beta s \mathcal{B}^{2} \mathbf{1}_{\mathbf{4}}}\left(\nabla_{i} \mathbf{1}_{\mathbf{4}}\right) e^{(1-\alpha-\beta) s \mathcal{B}^{2} \mathbf{1}_{4}} \\
T_{3}= & s^{2} \operatorname{Tr} \int_{0}^{1} d \alpha \int_{0}^{1-\alpha} d \beta e^{\alpha s \mathcal{B}^{2} \mathbf{1}_{4}}\left(\sigma_{\alpha \beta} F_{\alpha \beta}\right) \\
& \times e^{\beta s \mathcal{B}^{2} \mathbf{1}_{\mathbf{4}}}\left(\sigma_{\gamma \delta} F_{\gamma \delta}\right) e^{(1-\alpha-\beta) s \mathcal{B}^{2} \mathbf{1}_{4}}
\end{aligned}
$$

The terms $T_{1}$ and $T_{2}$ are of the same structure as in the gluon case and can be shown (see Appendix B of [18]) to yield two gauge invariant contributions:

$$
\begin{aligned}
I_{1}= & s^{3} \int_{0}^{1} d \alpha\left\{-\frac{1}{2}+\alpha(1-\alpha)+\frac{2}{9} s p^{2}\left[1-\frac{3}{2} \alpha(1-\alpha)\right]\right\} \\
& \times \operatorname{Tr} e^{(1-\alpha) s \mathcal{B}^{2}}\left\{\mathcal{B}, E_{i}\right\} e^{\alpha s \mathcal{B}^{2}}\left\{\mathcal{B}, E_{i}\right\} \mathbf{1}_{\mathbf{4}} \\
I_{2}= & -s^{2}\left(\frac{1}{2}-\frac{2}{9} s p^{2}\right) \operatorname{Tr} e^{s \mathcal{B}^{2}}\left(2 E_{i}^{2}+i\left\{\mathcal{B},\left[\nabla_{i}, E_{i}\right]\right\}\right) \mathbf{1}_{\mathbf{4}} \cdot(63)
\end{aligned}
$$


In contrast to the pure Yang-Mills calculation from [18] the Lorentz structure yields a factor 4 from $\operatorname{Tr} \mathbf{1}_{\mathbf{4}}$ and since we are dealing with fermions all matrices are in the fundamental representation. The second term in $I_{2}$ contains an anticommutator of $\mathcal{B}$ and the covariant divergence of the electric field, which is zero if the background field obeys the equation of motion. We will discuss it separately in the next section and leave it out for the time being.

What has to be evaluated is

$$
\begin{aligned}
& {\left[S_{1-\text { loop }}^{\mathrm{F}}\right]_{E}^{(2)}=-N_{f}\left[\log \operatorname{det}(i \not \nabla)_{n, r}\right]_{E}^{(2)}} \\
& =-\frac{N_{f}}{2} \int d^{3} x \sum_{k=-\infty}^{\infty} \int \frac{d^{3} p}{(2 \pi)^{3}} \int_{0}^{\infty} \frac{d s}{s} e^{-s p^{2}}\left(I_{1}+I_{2}+T_{3}\right) \\
& \equiv-N_{F}\left(L_{1}+L_{2}+L_{3}\right) .
\end{aligned}
$$

For the summation over the Matsubara frequencies it turns out to be necessary to define a region of definition for $\phi$. In particular we shall rescale this field as $\phi=2 \pi T \nu$ and look at the interval $-1 \leq \nu \leq 1$. In different regions of $\phi$ the results will have different functional forms. We already saw for the fermion potential that it is symmetric in the interval $-1 \leq \nu \leq 1$, and outside this region one has to continue analytically.

We will start with the term $T_{3}$. From

$$
\sigma_{\mu \nu}=-\frac{i}{4}\left[\gamma_{\mu}, \gamma_{\nu}\right]
$$

and

$$
\operatorname{Tr} \gamma_{\alpha} \gamma_{\beta} \gamma_{\gamma} \gamma_{\delta}=4\left(\delta_{\alpha \beta} \delta_{\gamma \delta}-\delta_{\alpha \gamma} \delta_{\beta \delta}+\delta_{\alpha \delta} \delta_{\beta \gamma}\right)
$$

it follows that

$$
\operatorname{Tr} \sigma_{\alpha \beta} \sigma_{\gamma \delta} F_{\alpha \beta} F_{\gamma \delta}=2 F_{\alpha \beta} F_{\alpha \beta} .
$$

Since $F_{\alpha \beta} F_{\alpha \beta}$ contributes to the electric sector as $2 E_{k} E_{k}$, eq. (61) is equal to

$$
\begin{aligned}
T_{3} & =4 s^{2} \operatorname{Tr} \int_{0}^{1} d \alpha \int_{0}^{1-\alpha} d \beta e^{\alpha s \mathcal{B}^{2}} E_{k} e^{\beta s \mathcal{B}^{2}} E_{k} e^{(1-\alpha-\beta) s \mathcal{B}^{2}} \\
& =2 s^{2} \operatorname{Tr} \int_{0}^{1} d \alpha e^{\alpha s \mathcal{B}^{2}} E_{k} e^{(1-\alpha) s \mathcal{B}^{2}} E_{k}
\end{aligned}
$$

where we used in the last line that the integrand is symmetric in $\alpha$ and $\beta$, invariant under $\alpha \rightarrow(1-\alpha)$ as well as the cyclicity of the trace. After integration over $\alpha, p$ and $s$ we find the following structure for $L_{3}$ :

$$
L_{3}=\frac{1}{4 \pi^{2}}\left[\left(\left(E_{i}^{1}\right)^{2}+\left(E_{i}^{2}\right)^{2}\right) \frac{\pi}{8} S_{1}+\left(E_{i}^{3}\right)^{2} \frac{\pi}{4} S_{3}\right],
$$

where

$$
\begin{aligned}
S_{1} & =\sum_{k=-\infty}^{\infty} \frac{\left|\phi+2 \omega_{k}\right|-\left|\phi-2 \omega_{k}\right|}{\phi \omega_{k}}=\frac{2}{\pi T}(\log 4 \mu) \\
S_{3} & =\sum_{k=-\infty}^{\infty}\left(\frac{1}{\left|\phi-2 \omega_{k}\right|}+\frac{1}{\left|\phi+2 \omega_{k}\right|}\right) \\
& =-\frac{1}{4 \pi T}\left[4\left(\gamma_{\mathrm{E}}-\log \mu\right)+\Phi(\nu)\right] .
\end{aligned}
$$

Here we used that $\omega_{k}=(2 k+1) \pi T$ and $\phi=2 \pi T \nu$. The function $\Phi(\nu)$ is given by

$$
\Phi(\nu)=2\left[\psi\left(\frac{1+\nu}{2}\right)+\psi\left(\frac{1-\nu}{2}\right)\right] .
$$

Here $\psi$ is the digamma function

$$
\psi(z)=\frac{\partial}{\partial z} \log \Gamma(z) .
$$

The parameter $\mu$ is the UV-cutoff in divergent series:

$$
\sum_{k=1}^{\infty} \frac{1}{k} \rightarrow \sum_{k=1}^{\mu} \frac{1}{k} \equiv \log \mu
$$

and is related to the Pauli-Villars mass as

$$
\mu=\frac{M}{4 \pi T} e^{\gamma_{\mathrm{E}}}
$$

This subtraction scale for the running coupling constant has been known previously [6] and was also obtained in [18].

Next we will turn to the invariant $I_{1}$. After integration over $\alpha, p$ and $s$ we find the following structure for $L_{1}$ :

$$
L_{1}=\frac{1}{4 \pi^{2}}\left[\left(\left(E_{i}^{1}\right)^{2}+\left(E_{i}^{2}\right)^{2}\right) \frac{\pi}{24} \tilde{S}_{1}+\left(E_{i}^{3}\right)^{2} \frac{\pi}{12} S_{3}(\nu)\right],
$$

where $S_{3}$ is given by eq. (71) and

$$
\begin{aligned}
\tilde{S}_{1}= & \sum_{k=-\infty}^{\infty} \frac{\left(\phi^{2}+4 \omega_{k}^{2}\right)}{\phi^{3} \omega_{k}} \\
& \times\left\{\frac{\phi^{2}-2 \phi \omega_{k}+4 \omega_{k}^{2}}{\left|\phi+2 \omega_{k}\right|}-\frac{\phi^{2}+2 \phi \omega_{k}+4 \omega_{k}^{2}}{\left|\phi-2 \omega_{k}\right|}\right\} \\
= & \frac{1}{\pi T}\left[2\left(\log \mu-\log 4-2 \gamma_{\mathrm{E}}\right)-\Phi(\nu)\right] .
\end{aligned}
$$

Finally we investigate $I_{2}$. Again after integration over $\alpha, p$ and $s, L_{2}$ is of the form:

$$
L_{2}=-\frac{1}{4 \pi^{2}}\left[\left(\left(E_{i}^{1}\right)^{2}+\left(E_{i}^{2}\right)^{2}+\left(E_{i}^{3}\right)^{2}\right) \frac{\pi}{6} S_{3}\right],
$$

with $S_{3}$ given by eq. (71).

Collecting all terms from $L_{1,2,3}$ we find the following results for the kinetic energy in the electric sector

$$
\begin{aligned}
{\left[S_{1-\text { loop }}^{\mathrm{F}}\right]_{E}^{(2)} } & =\int \frac{d^{3} x}{T}\left[\left(\left(E_{i}^{1}\right)^{2}+\left(E_{i}^{2}\right)^{2}\right) f_{1}^{(\mathrm{F})}(\nu)+\left(E_{i}^{3}\right)^{2} f_{3}^{(\mathrm{F})}(\nu)\right] \\
& =\int \frac{d^{3} x}{T}\left[E_{i}^{a} E_{i}^{a} f_{1}^{(\mathrm{F})}(\nu)+\frac{\left(E_{i}^{a} A_{4}^{a}\right)^{2}}{A_{4}^{b} A_{4}^{b}} f_{2}^{(\mathrm{F})}(\nu)\right], \quad(79)
\end{aligned}
$$

where $f_{2}^{(\mathrm{F})}(\nu) \equiv f_{3}^{(\mathrm{F})}(\nu)-f_{1}^{(\mathrm{F})}(\nu)$. In the second line of eq. (79) we used

$$
\left(E_{i}^{3}\right)^{2}=\frac{\left(E_{i}^{a} A_{4}^{a}\right)^{2}}{A_{4}^{b} A_{4}^{b}},
$$




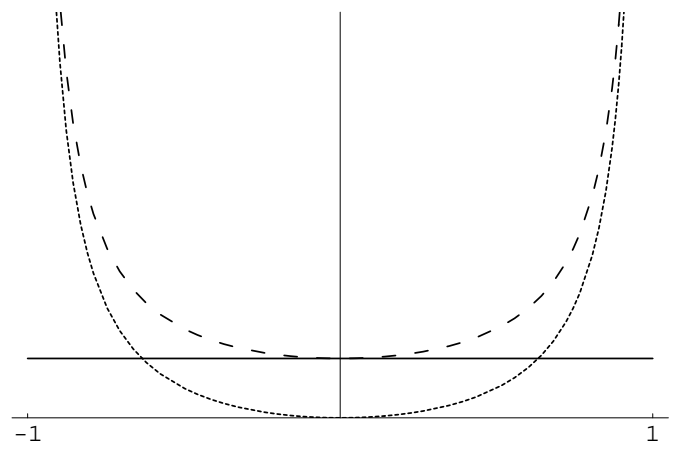

FIG. 2: The functions $f_{1}^{(\mathrm{F})}$ (solid), $f_{2}^{(\mathrm{F})}$ (dotted) and $f_{3}^{(\mathrm{F})}$ (dashed) without the UV divergent term.

which follows from eq. (41). The functions are given by

$$
\begin{aligned}
f_{1}^{(\mathrm{F})}(\nu) & =-\frac{N_{f}}{24 \pi^{2}} \log 4 \mu, \\
f_{3}^{(\mathrm{F})}(\nu) & =\frac{N_{f}}{96 \pi^{2}}\left[4\left(\gamma_{\mathrm{E}}-\log \mu\right)+\Phi(\nu)\right], \\
f_{2}^{(\mathrm{F})}(\nu) & =\frac{N_{f}}{96 \pi^{2}}\left[4\left(\gamma_{\mathrm{E}}+\log 4\right)+\Phi(\nu)\right] .
\end{aligned}
$$

We would like to stress once more that these functions are the results for the interval $-1 \leq \nu \leq 1$. They are plotted in Fig 2 and one sees that they are symmetric. To get outside the interval $-1 \leq \nu \leq 1$ one has to continue analytically, and the functional form of the $f_{i}^{(\mathrm{F})}$ changes.

We notice that the function $f_{1}^{(\mathrm{F})}$ is constant, i.e. independent of $A_{4}$. However, it contains the UV divergent $\log \mu$ that is necessary to renormalize the running coupling constant from the tree level action:

$$
-\frac{F_{\mu \nu}^{2}}{4 g^{2}(M)}=-\frac{F_{\mu \nu}^{2}}{8 \pi^{2}} \log \frac{M}{\Lambda}\left(\frac{11}{12} N_{c}-\frac{1}{6} N_{f}\right) .
$$

Here $N_{c}$ denotes the number of colors and $N_{f}$ the number of flavors. We correctly obtained the gluonic contribution to the charge renormalization in 18]. For the fermions the tree level divergence in the electric sector is

$$
\frac{E_{k} E_{k}}{2 g^{2}(M)}=E_{k} E_{k} \frac{N_{f}}{24 \pi^{2}} \log \frac{M}{\Lambda} .
$$

From our result eq. (81) we find the correct UV divergent contribution

$$
-\frac{E_{k} E_{k}}{24 \pi^{2}} \log \mu=-E_{k} E_{k} \frac{N_{f}}{24 \pi^{2}} \log \left(\frac{M}{4 \pi T} e^{\gamma_{\mathrm{E}}}\right) .
$$

If we add the tree-level and the 1-loop action then the result should be UV finite. This is obtained by choosing the scale $M$ in eq. (86) to be equal to the Pauli-Villars mass, which corresponds to the evaluation of the running coupling constant at the scale $4 \pi T / \exp \left(\gamma_{\mathrm{E}}\right)$. In the effective action we then have to replace the Pauli-Villars cutoff $M$ by $\Lambda$ and find

$$
\begin{aligned}
& F_{1}^{(\mathrm{F})}(\nu)=-\frac{N_{f}}{24 \pi^{2}} \log \frac{\Lambda}{4 \pi T} e^{\gamma_{\mathrm{E}}}, \\
& F_{3}^{(\mathrm{F})}(\nu)=\frac{N_{f}}{96 \pi^{2}}\left[-4 \log \frac{\Lambda}{4 \pi T}+\Phi(\nu)\right], \\
& F_{2}^{(\mathrm{F})}(\nu)=\frac{N_{f}}{96 \pi^{2}}\left[4\left(\gamma_{\mathrm{E}}+\log 4\right)+\Phi(\nu)\right]
\end{aligned}
$$

with $\Phi(\nu)$ given by eq. (72).

\section{The "equation of motion" term}

In the previous section we left away the contribution of the second term in eq. (63). Its contribution to the effective action is

$$
\begin{aligned}
S_{\mathrm{EM}} & =\frac{N_{f}}{2} \int d^{3} x \sum_{k=-\infty}^{\infty} \int \frac{d^{3} p}{(2 \pi)^{3}} \int_{0}^{\infty} \frac{d s}{s} e^{-s p^{2}} \\
& \times 4 s^{2}\left(\frac{1}{2}-\frac{2}{9} s p^{2}\right) \operatorname{Tr} e^{s \mathcal{B}^{2}}\left(i\left\{\mathcal{B},\left[\nabla_{i}, E_{i}\right]\right\}\right) .
\end{aligned}
$$

After integration and summation this becomes

$$
\begin{aligned}
S_{\mathrm{EM}} & =-\frac{N_{f}}{12 \pi} \int d^{3} x \frac{\operatorname{Tr}\left(\left[\nabla_{i}, E_{i}\right] A_{4}\right)}{\pi T} \\
& =-\frac{N_{f}}{12 \pi} \int d^{3} x \frac{\left(D_{i} E_{i}\right)^{a} A_{4}^{a}}{2 \pi T} .
\end{aligned}
$$

Here we wrote the result once in terms of a covariant derivative in the fundamental representation and once through a covariant derivative in the adjoint representation

$$
D_{i}^{a b}=\partial_{i} \delta^{a b}+f^{a c b} A_{i}^{c} \quad \text { and } \quad\left(D_{i} E_{i}\right)^{a}=D_{i}^{a e} E_{i}^{e},
$$

in order to compare to the gluon results [18. In [18] we obtained two terms: one comes solely from the non-zero Matsubara modes, while the other is the contribution of the zero mode alone. Our result here, eq. (91), is equal to $-N_{f} / 2$ times the first term of the gluon results.

Eq. (91) is zero if the background field obeys the equation of motion, $D_{i} E_{i}=0$. Otherwise it depends on the behavior of $A_{4}$ and $E_{i}$ at spatial infinity. One can integrate eq. (91) by parts and gets

$$
S_{\mathrm{EM}}=\frac{N_{f}}{24 \pi^{2} T} \int d^{3} x\left\{E_{i}^{a} E_{i}^{a}-\partial_{i}\left(E_{i}^{a} A_{4}^{a}\right)\right\}
$$

It yields a contribution to the function eq. 877) plus a full derivative term. There are certain background fields, BPS dyons [22] being an example, where $A_{4} \rightarrow$ const. and $E_{i} \sim \frac{1}{r^{2}}$ at spatial infinity. Therefore the full derivative term in eq. (931) is nonzero. However, in the particular case of the dyon, it satisfies the equation of motion and the two terms in eq. (93) cancel out. 


\section{Comparison to previous work}

In a related publication by Wirstam [28] an effective theory for QCD at high temperatures was derived by calculating gluon by gluon scattering at low momenta in terms of Feynman diagrams. In order to compare to the results of 28] we have to expand our functions (8789) to quadratic order in $\nu$. For eq. (87) this gives naturally zero, and the remaining contribution is

$$
-\int d^{3} x \frac{7 \zeta(3) N_{f}}{384 \pi^{4} T^{3}} A_{4}^{a} A_{4}^{a} E_{i}^{b} E_{i}^{b},
$$

which agrees with the result found in [28] if the gauge group is chosen to be $\mathrm{SU}(2)$.

\section{E. Leading terms in magnetic sector}

For an effective action in terms of magnetic fields we have to expand eq. (40) to quartic order in $\nabla_{i}$. The basic idea of the calculation is, again, to use master equations (5658) to drag covariant derivatives $\nabla_{i}$ to the right. One has for the commutators

$$
\begin{gathered}
\nabla_{i} e^{s \mathcal{B}^{2}}=e^{s \mathcal{B}^{2}} \nabla_{i}-i s \int_{0}^{1} d \delta e^{\delta s \mathcal{B}^{2}}\left\{\mathcal{B}, E_{i}\right\} e^{(1-\delta) s \mathcal{B}^{2}}, \\
\nabla_{i} \nabla_{j} e^{s \mathcal{B}^{2}}=e^{s \mathcal{B}^{2}} \nabla_{i} \nabla_{j}-i s \int_{0}^{1} d \delta e^{\delta s \mathcal{B}^{2}}\left[\nabla_{i} \nabla_{j}, \mathcal{B}^{2}\right] e^{(1-\delta) s \mathcal{B}^{2}}
\end{gathered}
$$

where

$$
\left[\nabla_{i} \nabla_{j}, \mathcal{B}^{2}\right]=-i \nabla_{i}\left\{\mathcal{B}, E_{j}\right\}-i\left\{\mathcal{B}, E_{i}\right\} \nabla_{j}
$$

In this way one ultimately obtains gauge-invariant combinations of the electric field in the fourth power, mixed terms containing both electric and magnetic fields, derivatives of the electric field and, finally, magnetic field squared. In this paper we restrict ourselves to the latter terms quadratic in the magnetic field $B_{i}$ defined as

$$
B_{i}=\frac{1}{2} \epsilon_{i j k} F_{j k}=B_{i}^{a} T^{a},
$$

where $F_{j k}=i\left[\nabla_{j}, \nabla_{k}\right]$ in the fundamental representation. For that reason we shall disregard the commutators $\left[\nabla_{i}, A_{4}\right]$ as they introduce powers of $E_{i}$. In addition, we restrict ourselves to the magnetic field parallel to $A_{4}$, i.e. $B_{i}=B_{i}^{3} T^{3}$. It means that we set the commutator $\left[F_{i j}, A_{4}\right]=i\left(\left[\nabla_{i}, E_{j}\right]-\left[\nabla_{i}, E_{j}\right]\right)$ to zero. In practical terms this means that we can drag all powers of the covariant derivative $\nabla_{i}$ as well as of the field strengths $F_{i j}$ through the exponentials of $A_{4}$, as if they commute. Looking at the argument of the exponent in eq. (40) we see that terms which are quadratic in $B_{i}$ either do not contain the field strength tensor $F_{\mu \nu}$ at all or consist only of powers of the latter. Mixing terms vanish upon integration over momentum. Hence similar to the gluon case (see Appendix C of [18]) we have to evaluate the following:

$$
\begin{aligned}
& {\left[S_{1-\text { loop }}^{\mathrm{F}}\right]_{M}^{(2)}=-N_{f}\left[\log \operatorname{det}(i \not \overline{ })_{n, r}\right]_{M}^{(2)}} \\
& =-\frac{N_{f}}{2} \int d^{3} x \sum_{k=-\infty}^{\infty} \int \frac{d^{3} p}{(2 \pi)^{3}} \int_{0}^{\infty} \frac{d s}{s} e^{-s p^{2}} \operatorname{Tr}\left[\left(V_{1}+V_{2}\right) \mathbf{1}_{\mathbf{4}}\right] \\
& \equiv-N_{f}\left[N_{1}-N_{2}\right],
\end{aligned}
$$

where $V_{1}$ only contains powers of the derivatives

$$
\begin{aligned}
V_{1} & =e^{s \mathcal{B}^{2}}\left(\frac{s^{2}}{2} \nabla^{2} \nabla^{2}+\frac{(2 i s)^{4}}{4 !} p_{i} p_{j} p_{k} p_{l} \nabla_{i} \nabla_{j} \nabla_{k} \nabla_{l}\right. \\
& \left.+\frac{(2 i s)^{2} s}{3 !} p_{i} p_{j}\left[\nabla^{2} \nabla_{i} \nabla_{j}+\nabla_{i} \nabla^{2} \nabla_{j}+\nabla_{i} \nabla_{j} \nabla^{2}\right]\right),
\end{aligned}
$$

and $V_{2}$ comes from the field strength tensor alone:

$$
\begin{aligned}
V_{2}= & s^{2} \operatorname{Tr} \int_{0}^{1} d \alpha \int_{0}^{1-\alpha} d \beta e^{\alpha s \mathcal{B}^{2}} \sigma_{i j} F_{i j} e^{\beta s \mathcal{B}^{2}} \\
& \times \sigma_{k m} F_{k m} e^{(1-\alpha-\beta) s \mathcal{B}^{2}}
\end{aligned}
$$

We start with the evaluation of $V_{1}$. For the momentum integration in we use

$$
\begin{aligned}
\int \frac{d^{3} p}{(2 \pi)^{3}} e^{-s p^{2}} & =\frac{1}{(4 \pi s)^{3 / 2}} \\
\int \frac{d^{3} p}{(2 \pi)^{3}} p_{i} p_{j} e^{-s p^{2}} & =\frac{1}{2 s} \frac{1}{(4 \pi s)^{3 / 2}} \delta_{i j} \\
\int \frac{d^{3} p}{(2 \pi)^{3}} p_{i} p_{j} p_{k} p_{m} e^{-s p^{2}} & =\frac{1}{(2 s)^{2}} \frac{1}{(4 \pi s)^{3 / 2}} \\
\times & {\left[\delta_{i j} \delta_{k m}+\delta_{i k} \delta_{j m}+\delta_{i m} \delta_{j k}\right] }
\end{aligned}
$$

and obtain the following contribution to eq. (96)):

$$
N_{1}=\frac{1}{4 \pi^{3 / 2}} \sum_{k=-\infty}^{\infty} \int_{0}^{\infty} \frac{d s}{\sqrt{s}} \operatorname{Tr} e^{s \mathcal{B}^{2}} \frac{1}{12}\left[\nabla_{i}, \nabla_{j}\right]\left[\nabla_{i}, \nabla_{j}\right]
$$

Since $\left[\nabla_{i}, \nabla_{j}\right]^{2}=-F_{i j} F_{i j}=-2 B_{k} B_{k}$, where $B_{k}=$ $B_{k}^{3} T^{3}$, this is equal to

$$
N_{1}=-\frac{1}{24 \pi^{3 / 2}} \sum_{k=-\infty}^{\infty} \int_{0}^{\infty} \frac{d s}{\sqrt{s}} \operatorname{Tr}\left(e^{s^{2} \mathcal{B}} B_{k} B_{k}\right),
$$

and after integration over $s$ and the summation over the Matsubara frequencies it becomes

$$
N_{1}=-\frac{1}{24 \pi^{3 / 2}} \int d^{3} x\left(B_{i}^{3}\right)^{2} \frac{\sqrt{\pi}}{2} S_{3},
$$

where $S_{3}$ is again given by eq. (171). For $V_{2}$ we find, using eqs. [66]67):

$$
V_{2}=2 s^{2} \operatorname{Tr} \int_{0}^{1} d \alpha \int_{0}^{1-\alpha} d \beta e^{\alpha s \mathcal{B}^{2}} F_{i j} e^{\beta s \mathcal{B}^{2}} F_{i j} e^{(1-\alpha-\beta) s \mathcal{B}^{2}} .
$$


Since the contribution to the magnetic sector of $F_{i j} F_{i j}$ is $2 B_{k} B_{k}$ this is equal to

$$
\begin{aligned}
V_{2} & =4 s^{2} \operatorname{Tr} \int_{0}^{1} d \alpha \int_{0}^{1-\alpha} d \beta e^{\alpha s \mathcal{B}^{2}} B_{k} e^{\beta s \mathcal{B}^{2}} B_{k} e^{(1-\alpha-\beta) s \mathcal{B}^{2}} \\
& =2 s^{2} \operatorname{Tr}\left(e^{s \mathcal{B}^{2}} B_{k} B_{k}\right)
\end{aligned}
$$

where we used the fact that the integrand is symmetric in $\alpha$ and $\beta$, the cyclic property of the trace and eventually dragged the magnetic field to the right. One hence sees that $V_{2}$ is of the same structure as $V_{1}$. Explicitly we find after the integrations over $p, s$ and the summation over $\omega_{k}$ that

$$
N_{2}=\frac{1}{16 \pi} \int d^{3} x\left(B_{i}^{3}\right)^{2} S_{3},
$$

where $S_{3}$ is given by eq. (71). Adding eqs. (104 107) we find the following result for the kinetic energy in the magnetic sector:

$$
\left[S_{1-\text { loop }}^{\mathrm{F}}\right]_{M}^{(2)} \int \frac{d^{3} x}{T}\left(B_{i}^{3}\right)^{2} h_{1}^{(\mathrm{F})}(\nu),
$$

where the coefficient is given by:

$$
h_{1}^{(\mathrm{F})}(\nu)=\frac{N_{f}}{96 \pi^{2}}\left[4\left(\gamma_{\mathrm{E}}-\log \mu\right)+\Phi(\nu)\right]
$$

with $\Phi(\nu)$ as in eq. (72).

The function $h_{1}^{(\mathrm{F})}$ above is the result for $-1 \leq \nu \leq$ 1 and it is symmetric in this interval. It also contains the necessary UV divergent contribution to cancel the tree-level divergence of the running coupling constant, eq. (84):

$$
-\frac{B_{k} B_{k}}{24 \pi^{2}} \log \mu=-\frac{B_{k} B_{k}}{24 \pi^{2}} \log \left(\frac{M}{4 \pi T} e^{\gamma_{\mathrm{E}}}\right) .
$$

Adding up the tree-level and 1-loop terms is obtained by replacing $\mu$ in eq. (109) by $\Lambda /(4 \pi T) \exp \left(\gamma_{\mathrm{E}}\right)$, which corresponds to an evaluation of the running coupling constant at the scale $4 \pi T / \exp \left(\gamma_{\mathrm{E}}\right)$. The final result is then:

$$
H_{1}^{(\mathrm{F})}(\nu)=\frac{N_{f}}{96 \pi^{2} T}\left[-4 \log \frac{\Lambda}{4 \pi T}+\Phi(\nu)\right] .
$$

\section{CONCLUSIONS}

We have calculated the 1-loop contribution of massless quarks to the effective action at high temperatures for any value of $A_{4}$ and hence of the Polyakov line. While we have a general result in the color-electric sector, we restrict ourselves to a magnetic field parallel to $A_{4}$. The covariant derivative expansion of this action has the form:

$$
\begin{aligned}
{\left[S_{\mathrm{eff}}^{\mathrm{F}}\right]^{(2)}=} & \int \frac{d^{3} x}{T}\left[-T^{3} V^{\mathrm{F}}(\nu)+E_{i}^{2} F_{1}^{(\mathrm{F})}(\nu)\right. \\
& \left.+\left(B_{i}^{\|}\right)^{2} H_{1}^{(\mathrm{F})}(\nu)+\ldots\right]
\end{aligned}
$$

where $\nu=\frac{\sqrt{A_{4}^{a} A_{4}^{a}}}{2 \pi T}$ and $B_{i}^{\|}$is the magnetic field parallel in color space to $A_{4}$. Because of the Bianchi identity, $\left[F_{i j}, A_{4}\right]=i\left(\left[\nabla_{i}, E_{j}\right]-\left[\nabla_{i}, E_{j}\right]\right)$, in the case where the magnetic field is not parallel to $A_{4}$ one also has to include terms with electric field and its derivatives into the effective action, otherwise it will not be complete.

The potential $V^{\mathrm{F}}$ has double the period as compared to the gluon induced potential, is symmetric in $\nu$ between -1 and 1 and has been known before. It has its minimum at $\left|A_{4}\right|=0$ and a maximum at $\left|A_{4}\right|= \pm 2 \pi T$. The functions $F_{1,2}^{(\mathrm{F})}$ and $H_{1}^{(\mathrm{F})}$ given by eqs. (87, 89) and eq. (111) are new. All functions, both in the electric and in the magnetic sector, are symmetric in $\nu$ between -1 and 1 , which reflects the fact, that fermions are in the fundamental representation of the color group. Our results can be used for studies of QCD at high but not infinite temperatures, where the Polyakov line experiences fluctuations which are large in amplitude but long ranged and where the dimensional reduction approach is too crude.

\section{Acknowledgments}

We are grateful to Chris Korthals-Altes for a critical reading of the manuscript and for helpful comments.

\section{Note added in proof}

After the submission, the paper by E. Megias, E. Ruiz Arriola and L.L. Salcedo, hep-ph/0312133 appeared on the net, in which the authors compute the coefficients in the parallel electric and magnetic sector. Apart from a constant our functions $h_{1}^{(\mathrm{F})}$ and $f_{3}^{(\mathrm{F})}$ agree with their results.

\section{APPENDIX A: EUCLIDEAN COORDINATES}

In finite temperature QCD one needs the Euclidean formulation of path integrals in order to give the partition function the statistical-mechanics interpretation. Superscripts $M$ will denote Minkowski coordinates, while superscripts $E$ will refer to Euclidean coordinates. Note that throughout the paper we have used Euclidean coordinates without any explicit superscripts.

For space-time coordinates we have

$$
x_{4}^{E}=i x_{0}^{M} \quad x_{i}^{E}=x_{i}^{M} .
$$

For the gluon and fermion fields we have

$$
\begin{array}{lr}
A_{4}^{E}=-i A_{0}^{M} & A_{i}^{E}=A_{i}^{M} \\
\psi_{f}^{E}=\psi_{f}^{M} & \psi_{f}^{\dagger E}=i \bar{\psi}_{f}{ }^{M} .
\end{array}
$$

The Dirac gamma matrices are related as:

$$
\gamma_{4}^{E}=\gamma_{0}^{M} \quad \gamma_{i}^{E}=-i \gamma_{i}^{M} \quad \gamma_{5}^{E}=\gamma_{5}^{M} .
$$

The Euclidean gamma matrices are hermitian. 
[1] A. M. Polyakov, Phys. Lett. B 72, 4770 (1978).

[2] A. D. Linde, Phys. Lett. B 96, 289 (1980).

[3] D. J. Gross, R. D. Pisarski and L. G. Yaffe, Rev. Mod. Phys.53, 43 (1981).

[4] P. Arnold and C. Zhai, Phys. Rev. D 50, 7603 (1994); ibid. 51, 1906 (1995); E. Braaten, Phys. Rev. Lett. 74, 2164 (1995); B. Kastening and C. Zhai, Phys. Rev. D 52, 7232 (1995); E. Braaten and A. Nieto, Phys. Rev. D 53, 3421 (1996).

[5] T. Appelquist and R.D. Pisarski, Phys. Rev. D 23, 2305 (1981); S. Nadkarni, Phys. Rev. D 27, 917 (1983); ibid. 38, 3287 (1988); N.P. Landsman, Nucl. Phys. B 322, 498 (1989).

[6] S. Huang and M. Lissia, Nucl. Phys. B 438, 54 (1995); K. Kajantie, M. Laine, K. Rummukainen and M. E. Shaposhnikov, Nucl. Phys. B 458, 90 (1996); ibid. 503, 357 (1997).

[7] E. Braaten and A. Nieto, Phys. Rev. D 51, 6990 (1995); P. Arnold and L.G. Yaffe, Phys. Rev. D 52, 7208 (1995); K. Kajantie, M. Laine, J. Peisa, A. Rajantie, K. Rummukainen and M.E. Shaposhnikov, Phys. Rev. Lett. 79, 3130 (1997); M. Laine and A. Rajantie, Nucl. Phys. B 513, 471 (1998); M. Laine and O. Philipsen, Nucl. Phys. B 523, 267 (1998);Phys. Lett. B 459, 259 (1999); K. Kajantie, M. Laine, A. Rajantie, K. Rummukainen and M. Tsypin, JHEP 9811, 011 (1998); A. Hart and O. Philipsen, Nucl. Phys. B 572, 243 (2000); A. Hart, M. Laine and O. Philipsen, Nucl. Phys. B 586, 443 (2000); K. Kajantie, M. Laine, K. Rummukainen and Y. Schroder, Phys. Rev. Lett. 86, 10 (2001); K. Kajantie, M. Laine and Y. Schroder, Phys. Rev. D 65, 045008 (2002); K. Kajantie, M. Laine, K. Rummukainen and Y. Schroder, Nucl. Phys. Proc. Suppl. 106, 525 (2002); K. Kajantie, M. Laine, K. Rummukainen and Y. Schroder, Phys. Rev. D 67, 105008 (2003).

[8] P. Arnold and L.G. Yaffe, Phys. Rev. D 57, 1178 (1998); D. Bodeker, Phys. Lett. B 426, 351 (1998); P. Arnold, D.T. Son and L.G. Yaffe, Phys. Rev. D 59, 105020 (1999); P. Arnold and L.G. Yaffe, Phys. Rev. D 62, 125013 (2000); P. Arnold, G. D. Moore and L.G. Yaffe, JHEP 0011, 001 (2000), ibid. 0301, 030 (2003), ibid.
0305, 051 (2003).

[9] E. Megias, E. Ruiz Arriola and L. L. Salcedo, Phys. Lett. B 563, 173 (2003); S. Leupold, hep-th/0302142

[10] C. P. Korthals-Altes, Nucl. Phys. B 420, 637 (1994).

[11] K. Fukushima and K. Ohta, J. Phys. G 26, 1397 (2000).

[12] L. Susskind, Phys. Rev. D 20, 2610 (1979).

[13] G. 't Hooft, Nucl. Phys. B 138, 1 (1978); ibid. 153, 141 (1979).

[14] B. Svetitsky and L. G. Yaffe, Nucl. Phys. B 210, 423 (1982).

[15] N. Weiss, Phys. Rev. D 24, 475 (1981).

[16] N. Weiss, Phys. Rev. D 25, 2667 (1982).

[17] V.M. Belyaev and V.L. Eletsky, JETP Lett. 50, 55 (1989), Pisma Zh.Eksp.Teor.Fiz. 50, 49 (1989); K. Enqvist and K. Kajantie, Z. Phys. C 47, 291 (1990).

[18] D. Diakonov and M. Oswald, Phys. Rev. D 68, 025012 (2003),

[19] M. Oswald, Acta Phys.Polon. B 34, 5847 (2003).

[20] D. Diakonov, V. Yu. Petrov and A. V. Yung, Phys. Lett. B 130, 385 (1983); Yad. Fiz. 39, 240 (1984); Sov. J. Nucl. Phys. 39, 150 (1984).

[21] B. Julia and A. Zee, Phys. Rev. D 11, 2227 (1975).

[22] M. K. Prasad and C. M. Sommerfield, Phys. Rev. Lett. 35, 760 (1975); E. B. Bogomol'nyi, Sov. J. Nucl. Phys. 24, 449 (1976).

[23] B. J. Harrington and H. K. Shephard, Phys. Rev. D 17, 2122 (1978); T. C. Kraan and P. van Baal, Phys. Lett. B 428, 268 (1998); Nucl. Phys. B 533, 627 (1998); K. Lee and C. Lu, Phys. Rev. D 58, 025011 (1998).

[24] O. Kaczmarek, S. Ejiri, F. Karsch, E. Laermann and F. Zantow, hep-lat/0312015 v2.

[25] J. D. Jackson and L. B. Okun, Rev. Mod. Phys.73, 663 (2001).

[26] J. Schwinger, Phys.Rev.82, 664 (1951).

[27] A. Hasenfratz and P. Hasenfratz, Phys. Lett. B 93, 165 (1980), Nucl. Phys. B 193, 210 (1981).

[28] J. Wirstam, Phys. Rev. D 65, 014020 (2002).

[29] Jackson and Okun [25] recommend to name the $\partial_{\mu} A_{\mu}=0$ gauge after the Dane Ludvig Lorenz and not after the Dutchman Hendrik Lorentz who certainly used this gauge too but several decades later. 